\title{
Lattice Gauge Theory Sum Rule for the Shear Channel
}

\author{
Harvey B. Meyer \\ Johannes Gutenberg-Universität Mainz, Institut für Kernphysik, D-55099 Mainz, Germany
}

(Dated: August 17, 2018)

\begin{abstract}
An exact expression is derived for the $(\omega, \boldsymbol{p})=0$ thermal correlator of shear stress in $\mathrm{SU}\left(N_{c}\right)$ lattice gauge theory. I remove a logarithmic divergence by taking a suitable linear combination of the shear correlator and the correlator of the energy density. The operator product expansion shows that the same linear combination has a finite limit when $\omega \rightarrow \infty$. It follows that the vacuum-subtracted shear spectral function vanishes at large frequencies at least as fast as $\alpha_{s}^{2}(\omega)$ and obeys a sum rule. The trace anomaly makes a potential contribution to the spectral sum rule which remains to be fully calculated, but which I estimate to be numerically small for $T \gtrsim 3 T_{c}$. By contrast with the bulk channel, the shear channel spectral density is then overall enhanced as compared to the spectral density in vacuo.
\end{abstract}

PACS numbers: 12.38.Gc, 12.38.Mh, 25.75.-q

\section{INTRODUCTION}

The shear viscosity $\eta$ is a quantity that universally characterizes the relaxation of a fluid towards equilibrium. Indeed $\eta c^{2} /\left[k_{B}(e+p)\right]$ is the diffusion coefficient of transverse momentum, and this process is in many situations occurring in Nature the dominant mechanism of dissipation ( $e$ is the energy density and $p$ the pressure of the system). The dimensionless quantity $\eta /(\hbar s)$ can be thought of as the ratio of a transport time scale $\eta /\left[k_{B}(e+p)\right]$ to the thermal time scale $\hbar /\left(k_{B} T\right)$ and is therefore a good measure of the ability of a fluid to flow [1, 2].

In the past years, the phenomenology of the RHIC heavy ion experiments has provided an upper bound on the ratio $\eta / s$ of hot quark matter (see [3] for a review; from here on we set $\hbar, c$ and $k_{B}$ to unity). In parallel to this, the thermodynamic properties of QCD in the range of temperatures $100 \lesssim T / \mathrm{MeV} \lesssim 700$ are the subject of ongoing Monte-Carlo simulations on a space-time lattice [46]. In this computational approach, access to the near-equilibrium properties, such as the shear viscosity, is limited because lattice QCD employs the Euclidean formulation of thermal field theory. Real-time properties can thus only be determined by analytic continuation, a numerically ill-posed

*Electronic address: meyerh@kph.uni-mainz.de 
problem (see e.g. [7], [8] section 5).

Through the Kubo formula, the shear viscosity is related to the low-frequency part of the spectral density, $\eta=\pi \lim _{\omega \rightarrow 0} \rho_{12,12} / \omega$ ([9] and Refs. therein). Here and in the following we denote by $\pi \rho_{\mu \nu, \rho \sigma}$ the imaginary part of the retarded correlator of the energy-momentum tensor components $T_{\mu \nu}$ and $T_{\rho \sigma}$. Some constraints on the thermal behavior of the spectral function have been obtained by computing the Euclidean correlator of the stress-energy tensor on the lattice [10, 11]. Because the spectral density is related by an integral transform to the Euclidean correlator, one is automatically led to studying the spectral density over the whole semi-axis of frequencies. For that reason, it is helpful to have global constraints on the spectral densities, such as sum rules [12, 13]. Very recently, I determined some of the gross features of the bulk-channel spectral density by combining Euclidean correlation functions with a spectral sum rule [14]. The goal of this paper is to take the steps necessary to apply the same strategy to the shear channel.

Romatschke and Son [15] obtained a shear sum rule for conformal field theories, and proposed a modified sum rule that takes into account the trace anomaly, $T_{\mu \mu}$. Here I give a derivation of the shear sum rule for the $\mathrm{SU}\left(N_{c}\right)$ gauge theory in the lattice regularization. In the continuum, the shear sum rule reads

$$
\int_{-\infty}^{\infty} \frac{\mathrm{d} \omega}{\omega}\left[\rho_{12,12}(\omega, \boldsymbol{p}=0, T)-\rho_{12,12}(\omega, \boldsymbol{p}=0,0)\right]=\frac{2}{3} e(T)-\lim _{\omega \rightarrow \infty} \Delta G(\omega, T),
$$

where $\Delta G(\omega, T)$ is defined by Eq. (31); I will show by explicit calculation that its $\omega \rightarrow \infty$ limit is a finite quantity, and that it is proportional to $e-3 p$. The right-hand side of Eq. (1) is therefore consistent with the form of the sum rule given by Romatschke and Son. Although the calculation of the coefficient remains to be completed, I estimate its practical importance for the determination of the shear viscosity in the range of temperatures explored at the LHC and find it to represent at most a $5 \%$ correction to the energy-density term on the right-hand side of Eq. (11). As a consequence, the right-hand side is positive at those temperatures, indicating that $\rho(\omega) / \omega$ is overall enhanced relative to the vacuum spectral density. This is in contrast with the bulk sum rule, which indicates an overall depletion of the bulk spectral weight throughout the deconfined phase.

I start by deriving an expression for the $\omega=\boldsymbol{p}=0$ Euclidean shear-channel correlator in section (III). Next I convert this identity into a spectral sum rule for the corresponding spectral density (Sec. III). The contact term that has to be subtracted before using the spectral representation is then shown to be finite in section (IV). I show that part of the finite term can be obtained from existing results, while the other remains to be calculated. The numerical importance of the contact term is estimated on the basis of this partial result. I make some final comments in section $(\bar{V})$. 


\section{DERIVATION OF THE LATTICE SUM RULE}

I start by introducing the essential notation, which follows closely [16]. The reader is referred to that paper for unexplained notation. The stress tensor in the $\mathrm{SU}\left(N_{c}\right)$ gauge theory reads $T_{\mu \nu}=\theta_{\mu \nu}+\frac{1}{4} \delta_{\mu \nu} \theta$,

$$
\theta(x) \equiv \beta(g) /(2 g) F_{\rho \sigma}^{a}(x) F_{\rho \sigma}^{a}(x) \quad \theta_{\mu \nu}(x) \equiv \frac{1}{4} \delta_{\mu \nu} F_{\rho \sigma}^{a} F_{\rho \sigma}^{a}-F_{\mu \alpha}^{a} F_{\nu \alpha}^{a} .
$$

The beta-function is defined by $q d g / d q=\beta(g)=-g^{3}\left(b_{0}+\ldots\right)$ and $b_{0}=11 N_{c} /\left(3(4 \pi)^{2}\right)$, $b_{1}=34 N_{c}^{2} /\left(3(4 \pi)^{4}\right)$ in the $\mathrm{SU}\left(N_{c}\right)$ pure gauge theory. If $\langle\ldots\rangle_{T}$ denotes the thermal average at temperature $T$,

$$
\epsilon-3 P=\langle\theta\rangle_{T}-\langle\theta\rangle_{0} \equiv\langle\theta\rangle_{T-0}, \quad \epsilon+P=\frac{4}{3}\left\langle\theta_{00}\right\rangle_{T}
$$

I will be considering the Wilson discretization of the $\mathrm{SU}\left(N_{c}\right)$ gauge theory on an anisotropic lattice,

$$
S_{\mathrm{g}}=\sum_{x} \beta_{\sigma} S_{\sigma}(x)+\beta_{\tau} S_{\tau}(x)
$$

where $S_{\sigma}$ and $S_{\tau}$ are respectively the sum of spatial and temporal plaquettes. The bare gauge coupling is given by $g_{0}^{2}=2 N_{c} / \sqrt{\beta_{\sigma} \beta_{\tau}}$. The time direction is discretized more finely by a factor $\xi$, called the (renormalized) anisotropy, than the spatial directions. I use the following discretizations,

$$
\begin{gathered}
\xi^{-3} \Theta(x)=Z_{\sigma}^{+}\left(\beta_{\sigma}, \beta_{\tau}\right) S_{\sigma}+Z_{\tau}^{+}\left(\beta_{\sigma}, \beta_{\tau}\right) S_{\tau} \\
\xi^{-3} \Theta_{00}(x)=Z_{\sigma}^{-}\left(\beta_{\sigma}, \beta_{\tau}\right) S_{\sigma}-Z_{\tau}^{-}\left(\beta_{\sigma}, \beta_{\tau}\right) S_{\tau} .
\end{gathered}
$$

The factor $Z_{\sigma, \tau}^{ \pm}$are such that $\left\langle\sum_{x} \Theta_{00}(x)\right\rangle \rightarrow\left\langle\int \mathrm{d}^{4} x \theta_{00}(x)\right\rangle$ in the continuum limit.

In the earlier publication [16], I derived lattice sum rules for the $(\omega, \boldsymbol{p})=0$ two-point functions of $\theta$ and $\theta_{00}$. In these sum rules, the functions of bare lattice parameters $\lambda_{00}^{+}$and $\lambda_{00}^{-}$appear, which were defined as

$$
\lambda_{00}^{ \pm}\left(\beta_{\sigma}, \beta_{\tau}\right) \equiv \frac{1}{2}\left(\frac{\partial}{\partial \beta_{\sigma}}-\frac{\partial}{\partial \beta_{\tau}}\right)\left(Z_{\sigma}^{-} \pm Z_{\tau}^{-}\right)
$$

In the appendix of [16], I gave the values of the latter in the limit $g_{0} \rightarrow 0$, corresponding to a lattice spacing exponentially smaller than the confinement scale. These values are wrong. The derivation of these values was based on the erroneous idea that the operator $\theta_{00}$ does not mix with the unit operator even on the anisotropic lattice. I presently correct this mistake. 


\section{A. Perturbative determination of $\lambda_{00}^{ \pm}$}

The parameters $\left(\beta_{\sigma}, \beta_{\tau}\right)$ appearing in the lattice action are related to the renormalized parameters $\left(a_{\sigma}, \xi\right)$ in a well-defined manner (up to $\mathrm{O}\left(a_{\sigma}^{2}\right)$ ambiguities; the spatial lattice spacing $a_{\sigma}$ is measured in units of a renormalized quantity such as a glueball mass). It is convenient to exchange the 'bare' variables $\left(\beta_{\sigma}, \beta_{\tau}\right)$ for $\left(\beta, \xi_{0}\right)$, where

$$
\xi_{0} \equiv \sqrt{\beta_{\tau} / \beta_{\sigma}}, \quad \text { and } \quad \beta \equiv \sqrt{\beta_{\sigma} \beta_{\tau}}
$$

The advantage of the new variables is that at the classical level, there is no difference between the bare anisotropy $\xi_{0}$ and the renormalized anisotropy $\xi$. Since the spatial and temporal plaquettes respectively yield $a_{\sigma}^{4} \boldsymbol{B}^{2}$ and $a_{\sigma}^{2} a_{\tau}^{2} \boldsymbol{E}^{2}$, it is not hard to guess the form of $Z_{\sigma, \tau}^{-}$,

$$
\begin{aligned}
& Z_{\sigma}^{-}=\frac{\beta}{\xi_{0}^{4}}=\frac{\beta_{\sigma}^{5 / 2}}{\beta_{\tau}^{3 / 2}}, \\
& Z_{\tau}^{-}=\frac{\beta}{\xi_{0}^{2}}=\frac{\beta_{\sigma}^{3 / 2}}{\beta_{\tau}^{1 / 2}},
\end{aligned}
$$

One then easily finds

$$
\begin{aligned}
& \lambda_{00}^{+}=3+\mathrm{O}\left(g_{0}^{2}\right), \\
& \lambda_{00}^{-}=1+\mathrm{O}\left(g_{0}^{2}\right) .
\end{aligned}
$$

To determine the quantum corrections, a little more work is needed. First, it was shown in [16] that at the isotropic point, the renormalization factors $Z_{\sigma, \tau}^{-}$are given by the renormalization of the anisotropy,

$$
Z_{\sigma}^{-\stackrel{\xi=1}{=}} Z_{\tau}^{-\stackrel{\xi=1}{=}} Z\left(g_{0}\right)=\left[\frac{\partial \xi_{0}\left(a_{\sigma}, \xi\right)}{\partial \xi}\right]_{\xi=1} .
$$

The factors $\lambda_{00}^{ \pm}$evaluated at the isotropic point can be written as

$$
\begin{aligned}
& \lambda_{00}^{-} \stackrel{\xi=1}{=} Z\left(g_{0}\right)+\frac{1}{\beta Z}\left[\frac{\partial^{2} \beta\left(a_{\sigma}, \xi\right)}{\partial(\log \xi)^{2}}-\frac{1}{16} \frac{d^{2} \beta\left(a_{\sigma}, \xi\right)}{d(\log a)^{2}}\right]_{\xi=1}, \\
& \lambda_{00}^{+} \stackrel{\xi=1}{=} 2+Z\left(g_{0}\right)-\frac{1}{Z\left(g_{0}\right)}\left[\frac{\partial^{2} \xi_{0}\left(a_{\sigma}, \xi\right)}{\partial \xi^{2}}\right]_{\xi=1}+\frac{g_{0}^{4}}{2 Z\left(g_{0}\right)} \frac{d g_{0}^{-2}}{d \log a} \frac{d Z\left(g_{0}\right)}{d g_{0}^{2}} .
\end{aligned}
$$

The relation between the bare and renormalized anistropy is known to one-loop order [17]. Indeed, two functions $c_{\sigma, \tau}(\xi)$ were computed there ([17], Eq. (2.24)-(2.25)), from which the coefficients $\left.c_{\sigma, \tau}^{\prime} \equiv \frac{d c_{\sigma, \tau}}{d \xi}\right|_{\xi=1}$ and $\left.c_{\sigma, \tau}^{\prime \prime} \equiv \frac{d^{2} c_{\sigma, \tau}}{d \xi^{2}}\right|_{\xi=1}$ can be obtained. The derivatives of the bare with respect to 
the renormalized anisotropy can be expressed in terms of these coefficients,

$$
\begin{aligned}
Z\left(g_{0}\right)=\left[\frac{d \xi_{0}}{d \xi}\right]_{\xi=1} & =1-\frac{1}{2}\left(c_{\sigma}^{\prime}-c_{\tau}^{\prime}\right) g_{0}^{2}+\mathrm{O}\left(g_{0}^{4}\right) \\
& {\left[\frac{d^{2} \xi_{0}}{d \xi^{2}}\right]_{\xi=1}=-g_{0}^{2}\left[c_{\sigma}^{\prime}-c_{\tau}^{\prime}+\frac{1}{2}\left(c_{\sigma}^{\prime \prime}-c_{\tau}^{\prime \prime}\right)\right]+\mathrm{O}\left(g_{0}^{4}\right) }
\end{aligned}
$$

For instance, numerically [17]

$$
c_{\sigma}^{\prime}-c_{\tau}^{\prime}=\frac{N_{c}^{2}-1}{N_{c}} \cdot 0.146711-N_{c} \cdot 0.019228
$$

Inspecting Eq. (14), we see that the square bracket is at least $\mathrm{O}\left(g_{0}^{2}\right)$, and therefore

$$
\lambda_{00}^{-}\left(g_{0}\right)=Z\left(g_{0}\right)+\mathrm{O}\left(g_{0}^{4}\right)=1-\frac{1}{2}\left(c_{\sigma}^{\prime}-c_{\tau}^{\prime}\right) g_{0}^{2}+\mathrm{O}\left(g_{0}^{4}\right)
$$

Similarly,

$$
\lambda_{00}^{+}\left(g_{0}\right)=3+\frac{g_{0}^{2}}{2}\left[c_{\sigma}^{\prime \prime}-c_{\tau}^{\prime \prime}+c_{\sigma}^{\prime}-c_{\tau}^{\prime}\right]+\mathrm{O}\left(g_{0}^{4}\right)
$$

These results will be used in sections (III) and (IV).

\section{B. Sum Rule for $\theta_{11}$}

In order to be sensitive to correlations of shear stress, we need to derive a sum rule for (say) the two-point function of the operator $\theta_{11}$. For that purpose we will need to work on an anisotropic lattice, and will take the isotropic limit at the end. To get at the $\theta_{11}$ correlator, the anisotropy has to be in the $\hat{1}$-direction.

Since it is conventional to associate the time-direction with the direction where the lattice spacing is smaller by a factor $\xi$, I temporarily interchange the labels of the $x$-direction $\hat{1}$ and the time-direction $\hat{0}$. I thus consider a lattice of dimensions $\infty_{0} \times\left(N_{\sigma} \times \infty_{2} \times \infty_{3}\right)$ and calculate the correlator of $\theta_{00}$ in this new coordinate system. At the end I will restore the normal labels, at what point the direction with $N_{\sigma}$ lattice points will play the role of the Matsubara cycle.

The procedure now closely follows [16]. First, consider a generic renormalization-group invariant quantity $f\left(a_{\sigma}, \xi, T\right)$, which is obtained as the continuum limit of a function of the bare parameters, $F\left(\beta_{\sigma}, \beta_{\tau}, N_{\sigma}\right)$. Expressing the independence of $f\left(a_{\sigma}, \xi, T\right)$ on $a_{\sigma}$ and $\xi$, I obtain respectively

$$
L \frac{\partial f}{\partial L}\left(\begin{array}{l}
1 \\
0
\end{array}\right)=\left(\begin{array}{cc}
\frac{\partial \beta_{\sigma}}{\partial \log a_{\sigma}} & \frac{\partial \beta_{\tau}}{\partial \log a_{\sigma}} \\
\frac{\partial \beta_{\sigma}}{\partial \log \xi} & \frac{\partial \beta_{\tau}}{\partial \log \xi}
\end{array}\right)\left(\begin{array}{c}
\frac{\partial F}{\beta_{\sigma}} \\
\frac{\partial F}{\beta_{\tau}}
\end{array}\right)
$$


At the symmetric point $\xi=1$, the determinant of the matrix is $2 \beta Z(\beta) \frac{d \beta}{d \log a}$. By taking a suitable linear combination, one finds

$$
-\frac{1}{4} L \frac{\partial f}{\partial L}=\beta Z(\beta)\left(\frac{\partial F}{\beta_{\sigma}}-\frac{\partial F}{\beta_{\tau}}\right)
$$

This equation holds up to $\mathrm{O}\left(a_{\sigma}^{2}\right)$ effects.

I now apply Eq. (22) to the case of

$$
f(L) \equiv L^{4}\left\langle\theta_{00}\right\rangle
$$

which is the continuum limit of the lattice expectation value

$$
F\left(\beta_{\sigma}, \beta_{\tau}, N_{\sigma}\right)=N_{\sigma}^{4} \xi\left(\left\langle\Theta_{00}\right\rangle_{L}-\left\langle\Theta_{00}\right\rangle_{(L=\infty)}\right) .
$$

Since we are on the anisotropic lattice, it is necessary to perform the subtraction on the infinite lattice $(L=\infty)$ in order to remove the mixing with the unit operator. After manipulations entirely similar to those performed in [16], I get

$$
\beta Z(\beta)\left(\frac{\partial F}{\partial \beta_{\sigma}}-\frac{\partial F}{\partial \beta_{\tau}}\right)=-4 F+N_{\sigma}^{4} \lambda_{00}^{-} \beta Z(\beta)\left\langle S_{+}\right\rangle_{\infty}^{L}+N_{\sigma}^{4} \lambda_{00}^{+}\left\langle\Theta_{00}\right\rangle_{\infty}^{L}-N_{\sigma}^{4} \sum_{x}\left\langle\Theta_{00}(x) \Theta_{00}(0)\right\rangle_{\infty}^{L} .
$$

Returning to the normal coordinate system, where the compact direction is time and $f$ is given by $-\frac{1}{4}(e+p)$ at the temperature $T=1 / L$, the lattice sum rule (22) can be written as

$$
a^{-4}\left\langle\sum_{x} \Theta_{11}(x) \Theta_{11}(0)\right\rangle_{T-0}-\frac{\beta Z(\beta) \lambda_{00}^{-}(\beta)}{\frac{d \beta}{d \log a}}(e-3 p)=\left(1-\frac{1}{4} \lambda_{00}^{+}\right)(e+p)+\frac{1}{16} T^{5} \partial_{T} \frac{e+p}{T^{4}} .
$$

For completeness I reproduce the sum rule for the $\theta_{00}$ correlator obtained in [16],

$$
a^{-4}\left\langle\sum_{x} \Theta_{00}(x) \Theta_{00}(0)\right\rangle_{T-0}-\frac{\beta Z(\beta) \lambda_{00}^{-}(\beta)}{\frac{d \beta}{d \log a}}(e-3 p)=\frac{3}{4} \lambda_{00}^{+}(\beta)(\epsilon+P)+\left(\frac{3}{4}\right)^{2} T^{5} \partial_{T} \frac{\epsilon+P}{T^{4}} .
$$

It is noteworthy that the difference of two-point functions

$$
\begin{aligned}
a^{-4} \sum_{x}\left\langle\frac{3}{4} \Theta_{11}(x) \Theta_{11}(0)\right. & \left.-\frac{3}{4} \Theta_{00}(x) \Theta_{00}(0)\right\rangle_{T-0} \\
& \equiv a^{-4} \sum_{x}\left\langle\frac{1}{4}\left(\Theta_{11}-\Theta_{22}\right)(x)\left(\Theta_{11}-\Theta_{22}\right)(0)-\frac{2}{3} \Theta_{00}(x) \Theta_{00}(0)\right\rangle \\
& =\frac{3}{4}\left(1-\lambda_{00}^{+}\right)(e+p)-\frac{3}{8} T^{5} \partial_{T} \frac{e+p}{T^{4}}
\end{aligned}
$$

is UV-finite. In writing Eq. (28) I have used the elementary identity

$$
\sum_{x}\left\langle\Theta_{00}(x) \Theta_{00}(0)\right\rangle=\sum_{x}\left\langle 3 \Theta_{11}(x) \Theta_{11}(0)+6 \Theta_{11} \Theta_{22}(0)\right\rangle .
$$

A potential application of Eq. (29) is a determination of the renormalization factor $Z\left(g_{0}\right)$, since it appears quadratically on the left-hand side and only linearly on the right-hand side of the equation. However it is probably in the continuum limit that this relation is most useful, as described in the next section. 


\section{SUM RULE IN THE CONTINUUM AND DISPERSION RELATION}

We are now ready to write a sum rule for the correlator of shear stress, $T_{12}$. I define

$$
\Delta G(\omega, T) \equiv \int \mathrm{d}^{4} x e^{i \omega x_{0}}\left\langle\frac{1}{4}\left(T_{11}(x)-T_{22}(x)\right)\left(T_{11}(0)-T_{22}(0)\right)-\frac{2}{3} T_{00}(x) T_{00}(0)\right\rangle_{T-0} .
$$

The lattice sum rule (29) shows that $\Delta G(0, T)$ is finite. In the next section, I will show that also $\lim _{\omega \rightarrow \infty} \Delta G(\omega, T)$ is UV-finite. I therefore write the dispersion relation for this correlator as (for the general method of deriving sum rules, see [15, 18])

$$
\begin{aligned}
\Delta G(0, T)-\lim _{\omega \rightarrow \infty} \Delta G(\omega, T) & =\int_{-\infty}^{\infty} \frac{\mathrm{d} \omega}{\omega}\left(\frac{1}{2} \Delta \rho_{11,11}(\omega, T)-\frac{1}{2} \Delta \rho_{11,22}(\omega, T)-\frac{2}{3} \Delta \rho_{00,00}(\omega, T)\right) \\
& =\int_{-\infty}^{\infty} \frac{\mathrm{d} \omega}{\omega} \Delta \rho_{12,12}(\omega, T)-\frac{2}{3} T c_{v}
\end{aligned}
$$

In the last step, I have used rotation symmetry, which implies $\rho_{12,12}=\frac{1}{2} \rho_{11,11}-\frac{1}{2} \rho_{11,22}$ (the spatial momentum $\boldsymbol{p}$ is set to zero throughout this section). I have also used the fact that $\rho_{00,00}(\omega, T)=$ $T c_{v} \omega \delta(\omega)$.

On the other hand, $\Delta G(0)$ can be rewritten identically as

$$
\Delta G(0, T)=\int \mathrm{d}^{4} x\left\langle\frac{3}{4} \theta_{11}(x) \theta_{11}(0)-\frac{3}{4} \theta_{00}(x) \theta_{00}(0)-\frac{1}{24} \theta(x) \theta(0)-\frac{1}{3} \theta_{00}(x) \theta(0)\right\rangle_{T-0} .
$$

In view of Eq. (29) and in view of the (continuum) sum rules [16]

$$
\begin{aligned}
\left\langle\int \mathrm{d}^{4} x \theta(x) \theta(0)\right\rangle_{T-0} & =T^{5} \partial_{T} \frac{e-3 p}{T^{4}} \\
\left\langle\int \mathrm{d}^{4} x \theta(x) \theta_{00}(0)\right\rangle_{T} & =\frac{3}{4} T^{5} \partial_{T} \frac{e+p}{T^{4}}
\end{aligned}
$$

we have, in infinite volume and in the continuum limit,

$$
\Delta G(0, T)=\frac{2}{3}\left(e-T c_{v}\right)
$$

To reach this expression, we have used the thermodynamic relations $c_{v}=\frac{\partial e}{\partial T}, s=\frac{\partial p}{\partial T}$, and $T s=$ $e+p$, as well as Eq. (11). Now combining Eq. (32) and (36), we reach Eq. (11) by recalling that, in infinite volume,

$$
\int \mathrm{d}^{3} x\left\langle\frac{1}{4}\left(T_{11}(x)-T_{22}(x)\right)\left(T_{11}(0)-T_{22}(0)\right)\right\rangle=\int \mathrm{d}^{3} x\left\langle T_{12}(x) T_{12}(0)\right\rangle .
$$

In the free theory, we can check the coefficient of the $e=\frac{d_{A} \pi^{2}}{15} T^{4}$ term in Eq. (1) $\left(d_{A} \equiv N_{c}^{2}-1\right)$. Using

$$
\rho_{12,12}^{\text {free }}(\omega, T)=\frac{d_{A}}{10(4 \pi)^{2}} \frac{\omega^{4}}{\tanh \omega / 4 T}+\left(\frac{2 \pi}{15}\right)^{2} d_{A} T^{4} \omega \delta(\omega)
$$

the coefficient of the energy density on the right-hand side of the sum rule is correctly reproduced. 


\section{CONTACT TERMS AND ASYMPTOTICS OF THE SPECTRAL DENSITY}

The spectral sum rule (11) contains the quantity $\lim _{\omega \rightarrow \infty} \Delta G(\omega, T)$. In this section we study the UV contact terms that appear in $\Delta G(\omega, T)$, since those are the only ones that survive in the infinite frequency limit. We are working in infinite volume and start by considering the contribution of the energy density correlator. At finite separation in time, this correlator has a simple time-independent expression,

$$
\int \mathrm{d}^{3} x\left\langle T_{00}(x) T_{00}(0)\right\rangle=T^{2} c_{v} \quad \forall x_{0} \neq 0
$$

The lattice sum rules, on the other hand, determine the correlator of $T_{00}$ integrated over all times. Combining these two pieces of information, one finds the contact term (see [16], section 3.3),

$$
\lim _{\omega \rightarrow \infty} \int \mathrm{d}^{4} x e^{i \omega x_{0}}\left\langle T_{00}(x) T_{00}(0)\right\rangle_{T-0}=\left(-\frac{1}{2 b_{0} g_{0}^{2}}+\text { finite }\right)(e-3 p)-\frac{3}{4}(e+p),
$$

where equation (12) has been used.

We now come to the contribution of the shear-stress correlator to $\Delta G$. At large frequencies, the operator-product expansion (OPE) is applicable. The leading-order result is [19] [26]

$$
\int \mathrm{d}^{4} x e^{i \omega x_{0}}\left\langle T_{12}(x) T_{12}(0)\right\rangle_{T-0} \stackrel{\omega \rightarrow \infty}{\sim}-\frac{1}{3 b_{0} g_{0}^{2}}(e-3 p)-\frac{1}{2}(e+p) .
$$

Thus we see that

$$
\Delta G(\omega, T) \stackrel{\omega \rightarrow \infty}{\sim} \mathrm{O}(1) \cdot(e-3 p)
$$

In particular, $\Delta G(\omega, T)$ is finite in the $\omega \rightarrow \infty$ limit, and its value comes entirely from the contribution of the $T_{\mu \mu}$ operator.

The fact that both the zero-frequency correlator $\Delta G(0, T)$ and the contact term $\lim _{\omega \rightarrow \infty} \Delta G(\omega, T)$ are finite implies that the correlator in coordinate space (i.e. as a function of Euclidean time $t$ ) diverges at short distances at most as

$$
\Delta G(t, T) \sim \frac{\alpha_{s}^{2}(1 / t)}{t} \cdot(\text { linear combination of } e, p)
$$

at small $t$. From the spectral representation of this correlator

$$
\Delta G(t, T) \stackrel{t \rightarrow 0}{\sim} \int_{\Omega}^{\infty} \mathrm{d} \omega\left(\Delta \rho_{12,12}(\omega, T)-\frac{2}{3} \Delta \rho_{00,00}(\omega, T)\right) e^{-\omega t},
$$

it follows that the leading behavior of the shear spectral function is at most

$$
\Delta \rho_{12,12}(\omega, T) \stackrel{\omega \rightarrow \infty}{\sim} \alpha_{s}^{2}(\omega) \cdot(\text { linear combination of } e, p)
$$


The leading order OPE result (41) does not allow us to obtain the finite part of the Wilson coefficient of the operator $T_{\mu \mu}$ in the OPE of $\lim _{\omega \rightarrow \infty} \Delta G(\omega, T)$. However, the lattice sum rules allow us to obtain the finite part of the $\left\langle T_{\mu \mu}\right\rangle_{T-0}=e-3 p$ coefficient in the OPE of the energy correlator [27],

$$
\lim _{\omega \rightarrow \infty} \int \mathrm{d}^{4} x e^{i \omega x_{0}}\left\langle T_{00}(x) T_{00}(0)\right\rangle_{T-0}=c_{\theta}(e-3 p)-\left(\frac{3}{4}+\mathrm{O}\left(g_{0}^{2}\right)\right)(e+p) .
$$

Indeed, the coefficient takes the value [16]

$$
c_{\theta}=\frac{\lambda_{00}^{-}\left(g_{0}\right) Z\left(g_{0}\right)}{g_{0}^{2} \frac{d g_{0}^{-2}}{d \log a}}-\frac{1}{4}+\mathrm{O}\left(g_{0}^{4}\right)=-\frac{1}{2 b_{0} g_{0}^{2}}+\frac{1}{2 b_{0}}\left(c_{\sigma}^{\prime}-c_{\tau}^{\prime}\right)+\frac{b_{1}}{2 b_{0}^{2}}-\frac{1}{4}+\mathrm{O}\left(g_{0}^{2}\right),
$$

where Eq. (19) has been used in the second equality. As shown above, the leading term, which diverges logarithmically in the lattice spacing, cancels in $\Delta G(\omega, T)$. The $\mathrm{O}\left(g_{0}^{2}\right)$ terms vanish in the continuum limit. For $N_{c}=3$, the finite terms in Eq. (47) numerically amount to, respectively,

$$
\lim _{g_{0} \rightarrow 0}\left(c_{\theta}+\frac{1}{2 b_{0} g_{0}^{2}}\right)=2.3942+0.4215-0.2500 \text {. }
$$

The dominant contribution comes from the regularization-dependent $\left(c_{\sigma}^{\prime}-c_{\tau}^{\prime}\right)$ term. It is associated with the lack of continuous translation invariance on the lattice. We expect the regularization dependence to cancel in $\Delta G(\omega, T)$, since it is a finite correlator. Therefore we take the two other terms to be representative of the size of $T_{\mu \mu}$ 's Wilson coefficient in the OPE of $\Delta G(\omega, T)$ (after the inclusion of a factor $\frac{2}{3}$, see Eq. (31)). In the $\mathrm{SU}(3)$ gauge theory for $T>2.5 T_{c},(e-3 p) / T^{4} \lesssim 0.5$ while $e / T^{4} \approx 4.5$ is very weakly temperature-dependent [20]. From the size of the Wilson coefficient $c_{\theta}$, we therefore expect $\lim _{\omega \rightarrow \infty} \Delta G(\omega, T)$ in the shear sum rule Eq. (11) to represent at most a $5 \%$ correction to the right-hand side of the equation.

\section{FINAL COMMENTS}

It is hoped that the spectral sum rule (1) will be useful in constraining the spectral density at finite temperature, especially when combined with lattice Monte-Carlo data on the Euclidean correlator. In order to be operational at all temperatures, the remaining contribution to $\Delta G(\omega, T)$ should be computed in the large-frequency limit. Since we have seen that one contribution to $\Delta G$ has a dependence on the regularization, it is important to compute $\Delta G$ as a whole in the same regularization. Dimensional regularization is then probably the computationally most economic choice.

The sum rule (1) is particularly useful in the pure gauge theory because the mass gap in the tensor channel is very large, $m_{2} / T_{c} \approx 7.9[21,22][28]$. Thus even at $T=3 T_{c}$, the substracted 
spectral density appearing in (1) coincides with the spectral density up to $\omega \approx 2.6 T$, in particular the latter is unaffected by the subtraction in the range of frequencies that determine the transport properties. Moreover, the contribution of the lightest tensor glueball to $\rho_{12,12}(\omega, \mathbf{0}, T=0)$ has been calculated on the lattice [22, 23]. It is parametrized by a matrix element $F_{T}$ in the notation of [23]. At $3 T_{c}$, this contribution to the spectral sum rule (1) is small,

$$
\frac{2}{\left(\frac{2 e\left(3 T_{c}\right)}{3}\right)} \int_{m_{2}-\epsilon}^{m_{2}+\epsilon} \frac{\mathrm{d} \omega}{\omega} \rho_{12,12}(\omega, \mathbf{0}, 0)=\frac{2 F_{T}^{2}}{m_{2}\left(\frac{2 e\left(3 T_{c}\right)}{3}\right)} \approx 2 \% .
$$

At high temperatures, there is another contribution to the spectral sum rule that can be clearly isolated and identified. For a weakly coupled field theory, where the spectral density admits a transport peak at the origin of width much smaller than temperature, Teaney derived a sum rule [24],

$$
\int_{-\Lambda}^{\Lambda} \frac{\mathrm{d} \omega}{\omega} \rho_{12,12}(\omega, \boldsymbol{p}, T) \approx \frac{1}{5}(e+p)\left\langle v_{\boldsymbol{p}}^{2}\right\rangle
$$

Here $\Lambda$ serves as a separation between the transport scale and the thermal scale. For ultra-

relativistic quasiparticles, as one might expect to find in the high-temperature gluon plasma, $\left\langle v_{\boldsymbol{p}}^{2}\right\rangle=$ 1. In that case, comparison of (50) with (1) reveals that at high temperatures, the spectral integral (11) receives an $\approx 40 \%$ contribution from the transport peak. This means in particular that the prospect of determining the area under the transport peak from a numerically determined Euclidean correlator is realistic.

\section{Acknowledgments}

I thank U. Wiedemann and J. Casalderrey-Solana for discussions at CERN, where this work was started.

[1] P. Kovtun, D. T. Son, and A. O. Starinets, Phys. Rev. Lett. 94, 111601 (2005), hep-th/0405231.

[2] L. P. Csernai, J. I. Kapusta, and L. D. McLerran, Phys. Rev. Lett. 97, 152303 (2006), nucl-th/0604032.

[3] D. A. Teaney (2009), 0905.2433.

[4] Y. Aoki et al., JHEP 06, 088 (2009), 0903.4155.

[5] A. Bazavov et al., Phys. Rev. D80, 014504 (2009), 0903.4379.

[6] M. Cheng et al., Phys. Rev. D81, 054504 (2010), 0911.2215.

[7] G. Cuniberti, E. De Micheli, and G. A. Viano, Commun. Math. Phys. 216, 59 (2001).

[8] A. Bazavov, P. Petreczky, and A. Velytsky (2009), 0904.1748. 
[9] D. T. Son and A. O. Starinets, Ann. Rev. Nucl. Part. Sci. 57, 95 (2007), 0704.0240.

[10] H. B. Meyer, Phys. Rev. D76, 101701 (2007), 0704.1801.

[11] H. B. Meyer, Phys. Rev. Lett. 100, 162001 (2008), 0710.3717.

[12] D. Kharzeev and K. Tuchin, JHEP 09, 093 (2008), 0705.4280.

[13] F. Karsch, D. Kharzeev, and K. Tuchin, Phys. Lett. B663, 217 (2008), 0711.0914.

[14] H. B. Meyer, JHEP 04, 099 (2010), 1002.3343.

[15] P. Romatschke and D. T. Son, Phys. Rev. D80, 065021 (2009), 0903.3946.

[16] H. B. Meyer, Nucl. Phys. B795, 230 (2008), 0711.0738.

[17] F. Karsch, Nucl. Phys. B205, 285 (1982).

[18] J. I. Kapusta and E. V. Shuryak, Phys. Rev. D49, 4694 (1994), hep-ph/9312245.

[19] S. Caron-Huot, Phys. Rev. D79, 125009 (2009), 0903.3958.

[20] G. Boyd et al., Nucl. Phys. B469, 419 (1996), hep-lat/9602007.

[21] H. B. Meyer and M. J. Teper, Phys. Lett. B605, 344 (2005), hep-ph/0409183.

[22] Y. Chen et al., Phys. Rev. D73, 014516 (2006), hep-lat/0510074.

[23] H. B. Meyer, JHEP 01, 071 (2009), 0808.3151.

[24] D. Teaney, Phys. Rev. D74, 045025 (2006), hep-ph/0602044.

[25] V. A. Novikov, M. A. Shifman, A. I. Vainshtein, and V. I. Zakharov, Fortschr. Phys. 32, 585 (1984).

[26] I have checked the results using the methods of [25].

[27] The large-frequency limit is taken in the regime $\omega \ll \frac{\pi}{a}$. Equivalently, the contact term is defined via a separation distance scale $d$, with $a \ll d \ll T^{-1}$, by the difference $\left(\int_{0}^{1 / T}-\int_{d}^{1 / T-d}\right) \mathrm{d} x_{0} \int \mathrm{d}^{3} \boldsymbol{x}\left\langle T_{00}(x) T_{00}(0)\right\rangle_{T-0}$. For instance, one could scale $d$ as $(a T)^{\frac{1}{2}}$.

[28] Or $m_{2} \approx 2.4 \mathrm{GeV}$ if one sets the scale with the Sommer parameter $r_{0}^{-1}=0.41 \mathrm{GeV}$ 